\title{
Diagnose und Differenzialdiagnose des Cushing-Syndroms - Fall 3/2015
}

\author{
B. Besemer, J. Honegger, A. Bornemann, P. Adam, M. Horger, C. Maser-Gluth, K. Müssig
}

Beim Cushing-Syndrom produziert der Körper zu viel Kortisol oder erhält zu viele synthetische Glukokortikoide. Die Diagnose des Hyperkortisolimus ist anhand der Anamnese und klinischen Symptome meist rasch gestellt. Aufgrund der Bandbreite an möglichen Ursachen ist die Differenzialdiagnostik jedoch alles andere als trivial. Lesen Sie hier in Kurzform zwei Fälle von Patientinnen mit Cushing-Syndrom und erfahren Sie mehr über die vielfältigen Ursachen. Den ausführlichen „Tübinger Fall“ mit allen Details zu den Patientinnen und Untersuchungen, vielen Abbildungen und den Hintergründen finden Sie im Internet.

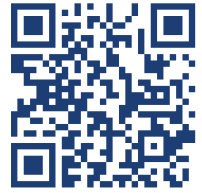

QR-Code einscannen und kompletten Fall online lesen oder unter:

http://dx.doi.org/ $10.1055 / \mathrm{s}-0041-103503$

\section{Hormoneller Regelkreis}

Bildung in der Nebennierenrinde I Die KortisolProduktion in der Nebennieren wird über die hypothalamisch-hypophysäre Achse stimuliert:

- Kortikotropin-Releasing-Hormon (CRH): im Hypothalamus gebildet; stimuliert Hypophyse

- adrenokortikotropes Hormon (ACTH): in Hypophyse gebildet; stimuliert Nebennierenrinde Kortisol und ACTH hemmen wiederum die CRHSekrektion.

Zirkadiane Rhythmik | Die Kortisol-Ausschüttung unterliegt einer ausgesprochenen zirkadianen Rhythmik. Dabei werden die höchsten Werte am frühen Morgen und die niedrigsten gegen Mitternacht verzeichnet.

\section{Ursachen des Cushing-Syndroms}

latrogen I Meist entsteht das Cushing-Syndrom iatrogen durch eine Langzeittherapie mit Glukokortikoiden, z. B. bei

- chronisch entzündlichen Darmerkrankungen,

- chronische Bronchitis (COPD) und

- rheumatischen Erkrankungen.
Abb. 1 Klassische Stigmata des Hyperkortisolismus: „Vollmondgesicht“ und Gesichtsplethora (A), Büffelnacken und supraklavikuläre Fettpolster (B).

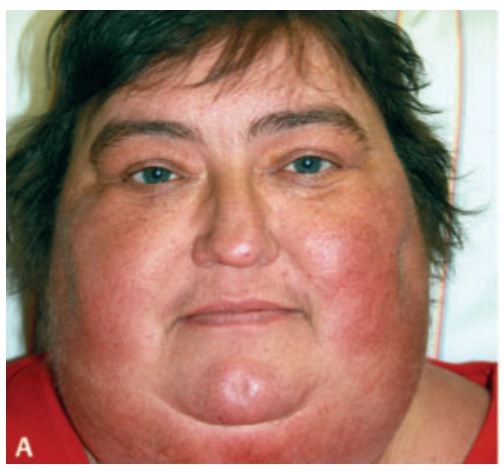

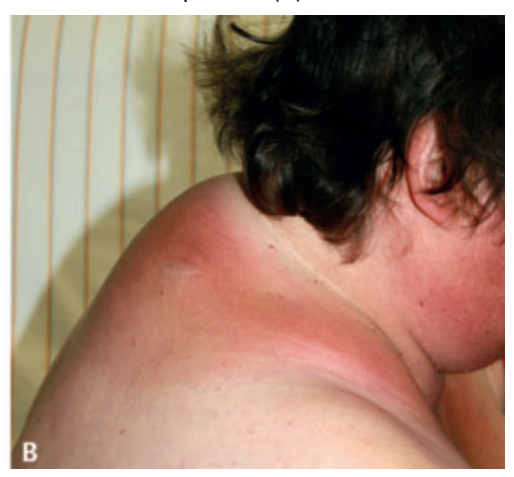

ACTH-abhängig I In 85\% der Fälle eines endogenen Hyperkortisolismus liegt ein ACTH-abhängiges Cushing-Syndrom vor. Zwei Formen werden unterschieden:

- zentral/hypophysär (Morbus Cushing; 80\%): meist bedingt durch ein Hypophysenadenom, selten -karzinom

- ektop (paraneoplastisch; 20\%): z.B. bei Bronchialkarzinom oder Bronchuskarzinoid

Cave Aufgrund des schnellen Tumorwachstums bilden sich bei einem kleinzelligen Bronchialkarzinom die klinischen Stigmata des Hyperkortisolismus häufig nicht aus.

ACTH-unabhängig I 15\% der Fälle eines endogenen Hyperkortisolismus werden ACTH-unabhängig von Adenomen bzw. Karzinomen der Nebennierenrinde verursacht (jeweils 50\%).

\section{Kasuistik 1: 50-jährige Patientin}

Anamnese | Eine 50-jährige Frau berichetet über eine Gewichtszunahme von $15 \mathrm{~kg}$ in den letzten 3 Monaten bei zunehmender Verschlechterung des Allgemeinzustands. Vorerkrankungen:

- arterielle Hypertonie

- Diabetes mellitus Typ 2 (orale Antidiabetika)

- Asthma bronchiale (u.a. 7,5 mg/d Prednisolon)

Klinischer Befund I Der Allgemeinzustand der Patientin war deutlich reduziert, der Ernährungszustand adipös (BMI 60,2 kg/ $\mathrm{m}^{2}$ ).

- Puls: 125 Schläge/min

- Blutdruck: 175/95 mmHg

Es imponierten die klassischen Stigmata des Hyperkortisolismus ( Abb. 1).

Laboruntersuchungen I Der niedrigdosierte Dexamethsonhemmtest und die Bestimmung von Mitternachts-Serumkortisol und freiem Kortisol 
im 24-h-Sammelurin sprachen für einen Hyperkortisolismus. Die Differenzialdiagnostik ergab einen Morbus Cushing.

Bildgebung| Im Schädel-MRT zeigte sich intraund suprasellär eine Raumforderung mit einer Ausdehung von $10 \times 5 \times 12 \mathrm{~mm}$, die kurz darauf operativ entfernt wurde.

Histologie | Die Histologie bestätigte die Verdachtsdiagnose eines Hypophysenadenoms.

Verlauf | Aufgrund von postoperativ verminderten Serumkortisolwerten wurde vorübergehend eine Substitution mit Hydrocortison begonnen. Der weitere postoperative Verlauf war unauffällig.

\section{Kasuistik 2: 27-jährige Patientin}

Anamnese | Eine 27-jährige Frau berichetet über eine Gewichtszunahme von $4 \mathrm{~kg}$ in den letzten 2 Monaten bei Verschlechterung des Allgemeinzustands mit Adynamie und Muskelschwäche. Die Patientin klagte zudem über

- eine sekundäre Amenorrhoe,

- Schlafstörungen und

- eine Hämatomneigung.

Keine relevanten Vorerkrankungen oder regelmäßige Medikamenteneinnahme.

Klinischer Befund | Der Allgemeinzustand der Patientin war reduziert, der Ernährungszustand normal (BMI $24,1 \mathrm{~kg} / \mathrm{m}^{2}$ ).

- Puls: 100 Schläge /min

- Blutdruck: 150/100 mmHg

Die klassischen Stigmata des Hyperkortisolismus waren ebenfalls deutlich zu erkennen.

Laboruntersuchungen I Auch hier ergaben die Screening-Tests einen Hyperkortisolismus. Darüberhinaus waren androgene, mineralo- und glukokortikoide Vorstufen erhöht. Die weitere Differenzialdiagnostik ergab ein ACTH-unabhängiges Cushing-Syndrom.

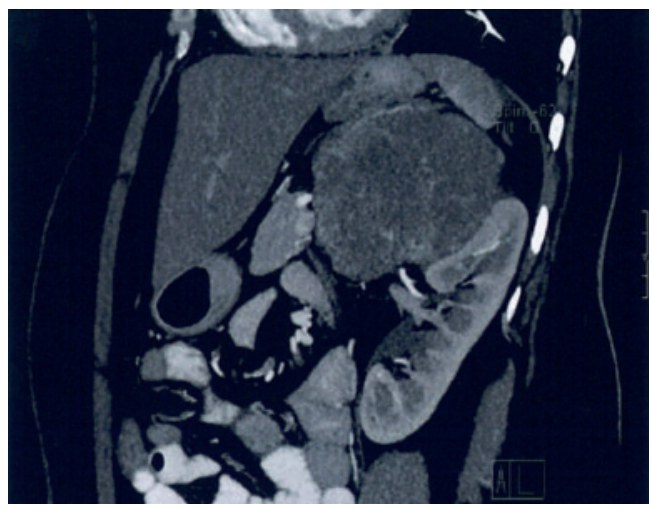

Abb. 2 Raumforderung der linken Nebenniere $(91 \times 65 \mathrm{~mm})$.
Bildgebung I Nun erfolgte eine Abdomensonografie sowie ein Abdomen-CT. Es zeigte sich eine inhomogene, weichteiläquivalente Raumforderung der linken Nebenniere der Größe $91 \times 65 \mathrm{~mm}$ ( Abb. 2). Sie wurde operativ entfernt.

Histologie I Es handelte sich bei dem Tumor um ein Nebennierenrindenkarzinom (Initialstadium nach Bradley: T2, M0, R1, G2).

Verlauf I 10 Tage nach der Operation hatten sich die Laborparameter normalisiert. Ein postoperatives Thorax-CT zeigte mehrere Lungenmetastasen. Die Therapie wurde multimodal fortgeführt. Nach mehrmaligem Progress starb die Patientin infolge einer kardialen und respiratorischen Insuffizienz. Seit der Erstdiagnose waren 14 Monate vergangen.

\section{Konsequenz für Klinik und Praxis}

- Bei Vorliegen klinischer Zeichen des Hyperkortisolismus sollte eine rasche Diagnostik eingeleitet werden.

- Nur eine umfassende Differenzialdiagnostik ermöglicht bei endogenem Hyperkortisolismus eine individuelle Therapie .

- An erster Stelle steht die Labordiagnostik, ergänzt durch bildgebende Verfahren je nach Verdachtsdiagnose.
Korrespondenz

Prof. Dr. med.

Karsten Müssig

Institut für Klinische

Diabetologie

Deutsches Diabetes-Zentrum Auf'm Hennekamp 65 40225 Düsseldorf

E-Mail: karsten.muessig@ddz. uni-duesseldorf.de

DOI 10.1055/s-0041-103503 Dtsch Med Wochenschr 2015; 140: 1294-1295 (c) Georg Thieme Verlag KG Stuttgart · New York. ISSN 0012-0472

\section{Die Tübinger Fälle in der DMW}

- Die Tübinger Fälle sind Fallbeispiele aus der Universitätsklinik Tübingen. Die Kasuistiken sind didaktisch speziell aufbereitet und enthalten viele interaktive Elemente.

- Die kompletten Fälle im PDF-Format können Sie bequem online ansehen. Schritt für Schritt schildern die Autoren Anamnese, Untersuchungen, Befundung und Therapie.

- Im Anschluss an die Kasuistik diskutieren sie ihre Vorgehensweise anhand aktueller Literaturdaten.

- Zum kompletten Tübinger Fall gelangen Sie im Internet unter http://dx.doi.org/10.1055/s-0041-103503

- Eine Auflistung aller bisher erschienen Tübinger Fälle finden Sie unter www.thieme.de/dmw

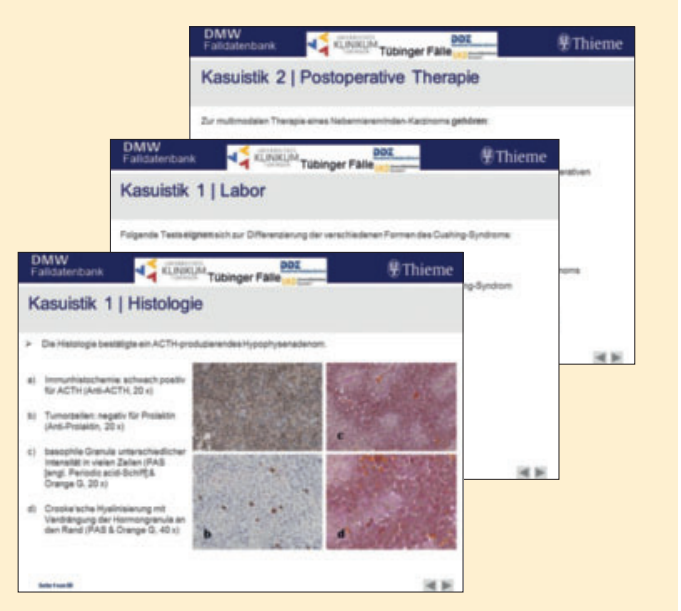

